\title{
The liver fluke Opisthorchis felineus as a group III or group I carcinogen
}

\author{
Mariya Yurievna Pakharukova, ${ }^{1,2}$, José Manuel Correia da Costa ${ }^{3,4}$, and Viatcheslav Alekseevitch Mordvinov ${ }^{1, *}$ \\ ${ }^{1}$ Laboratory of Molecular Mechanisms of Pathological Processes, Institute of Cytology and Genetics, Siberian Branch of Russian \\ Academy of Sciences, 10 Lavrentiev Avenue, 630090 Novosibirsk, Russia \\ ${ }^{2}$ Novosibirsk State University, 2 Pirogova Street, 630090 Novosibirsk, Russia \\ ${ }^{3}$ Center for the Study in Animal Science, ICETA, University of Porto, Rua de D. Manuel II, Apt 55142, 4051-401 Porto, Portugal \\ ${ }^{4}$ Centre for Parasite Immunology and Biology, Infectious Diseases Department, INSA, Rua Alexandre Herculano 321, 4000-055 \\ Porto, Portugal
}

Received 31 October 2018, Accepted 10 May 2019

\begin{abstract}
Opisthorchiasis caused by the liver fluke Opisthorchis felineus is one of the most common helminthic infections in the Russian Federation. The largest area affected by opisthorchiasis felinea occupies almost the entire territory of Western Siberia and extends to northern Kazakhstan and a part of the Ural region. Natural endemic regions of opisthorchiasis also exist in the European part of Russia, and in the regions of Western and Eastern Europe. According to the official statistics of the Russian Federation, up to 40000 patients with opisthorchiasis are registered annually in the country.

Opisthorchiasis felinea affects the hepatobiliary system and causes serious liver disorders, including cancer of the biliary tract. Other parasitoses, opisthorchiasis viverrini and clonorchiasis, are widespread in the Southeast Asia and China. The causative agents of these diseases, liver flukes O. viverrini and Clonorchis sinensis, are officially recognized as Group 1 biological carcinogens and are classified as the main risk factors for cholangiocarcinoma. O. felineus is included in Group 3 of biological carcinogens and is not officially considered carcinogenic to humans. Studies on the carcinogenic potential of this liver fluke and the epidemiology of cholangiocarcinoma in the Russian Federation have started in earnest quite recently. Nevertheless, we have some evidence that infection with $O$. felineus leads to a precancerous state of the bile duct epithelium. This state, combined with additional risk factors, poses a real risk of cholangiocarcinoma. In our opinion, taking into consideration the accumulated facts, the classification of the carcinogenic potential of $O$. felineus requires revision. In this review, we focus on the relevant characteristics of the biology and epidemiology of this helminth as well as experimental data on opisthorchiasis felinea; this information might clarify the carcinogenicity of O. felineus to humans.
\end{abstract}

Keywords: Cancer, Carcinogenic, Carcinogenesis, Carcinoma, Cell transition, Cercaria, Cholangiocellular carcinoma, Clonorchis, Cyprinidae, Epidemiology, Fish, Helminthiasis, Hepatocellular carcinoma, Liver flukes, Malignancy, Opisthorchiasis, Opisthorchis felineus, Pathogenesis, Risk, Sporocysts, Tumor

\section{Introduction}

Opisthorchis felineus (O. felineus) is a foodborne liver fluke, a member of the genus Opisthorchis, family Opisthorchiidae, order Digenea, class Trematoda, phylum Platyhelminthes, and kingdom Animalia. This liver fluke is a causative agent of opisthorchiasis felinea, a helminthiasis affecting mainly the hepatobiliary system and pancreas. Infection occurs after consumption of raw or undercooked freshwater fish infested with helminth larvae. Characteristics of the human disease are: long duration, frequent

\footnotetext{
*Corresponding author: mordvin@bionet.nsc.ru
}

exacerbations, and possible induction of primary liver cancer [1-6].

Liver flukes O. viverrini and Clonorchis sinensis, also members of the family Opisthorchiidae, cause opisthorchiasis viverrini and clonorchiasis, the diseases that are very similar to opisthorchiasis felinea in symptomatology and pathophysiology [7-10]. Opisthorchiasis and clonorchiasis are trematodiases whose natural hot spots cover a considerable part of Europe and Asia. Nonetheless, currently, these diseases might be arbitrarily attributed to natural focal infections. Many migrants from Asia live in the nonendemic areas of opisthorchiasis and clonorchiasis, and 
the tourist traffic among various countries is steadily increasing. Consequently, patients with liver fluke infection can be registered far from the corresponding endemic regions [6].

The International Agency for Research on Cancer (IARC) has classified liver flukes $O$. viverrini and $C$. sinensis as Group 1 agents, i.e., the agents carcinogenic to humans [7] and as the major causative factors of cholangiocarcinoma (CCC) in endemic regions [7-9]. CCC with features of cholangiocyte differentiation is one of the main histological types of malignant tumors of biliary-tract epithelia and is a relatively rare type of liver cancer [10]. Nevertheless, over the last 15 years, its incidence has steadily increased worldwide, and nowadays, it represents the second most common type of primary liver cancer (15-20\% of cases) after hepatocellular carcinoma [11]. The only available therapy for CCC is surgical resection or a liver transplant. Usually, CCC is diagnosed at advanced stages and is regarded as an incurable and lethal cancer with a poor survival rate of $<24$ months [12]. Both experimental and epidemiological evidence strongly implicate liver fluke infection in the etiology of CCC [13].

Opisthorchis felineus is still officially classified as a Group 3 carcinogen; this group includes factors not classified as carcinogenic to humans [7]. Meanwhile, data have been accumulating that show the carcinogenic potential of O. felineus and support its classification as a human biological carcinogen. This review summarizes the data indicating that $O$. felineus is an underestimated parasite, and that there is a need to revisit and revise the status of this liver fluke.

\section{Biology}

The complex life cycle of $O$. felineus includes the first and second intermediate hosts and one final host (Fig. 1) $[14,15]$. By getting into water containing the feces of humans and animals, eggs can be eaten by aquatic, gastropod snail of the genus Bithynia. In their intestines, mobile larvae (miracidia) hatch from the eggs, penetrate through the intestinal wall into the body cavity of the mollusk, and turn into sporocysts. The latter give birth to rediae, which can then produce cercariae: free-living motile larvae that enter water, where they actively swim. The parthenogenetic phase in the life cycle of the helminth (from the stage of sporocysts to the release of cercaria) lasts $\sim 2$ months. Development is hampered by the diapause of the host during the winter period (freezing of rivers) in Western Siberia. The first cercariae appear in May of the following year. Mature cercariae are motile and begin to emerge from mollusks in bright sunny weather at a rate of up to several hundred a day [15].

Freshwater fish susceptible to opisthorchiid infestation are Cyprinidae species: ide Leuciscus idus, dace L. leuciscus, roach Rutilus rutilus, and sunbleak Leucaspius delineatus $[14,15]$. When fish and cercariae encounter each other, cercariae attach to them, discard the tail and penetrate through the skin into the muscles, where they form the

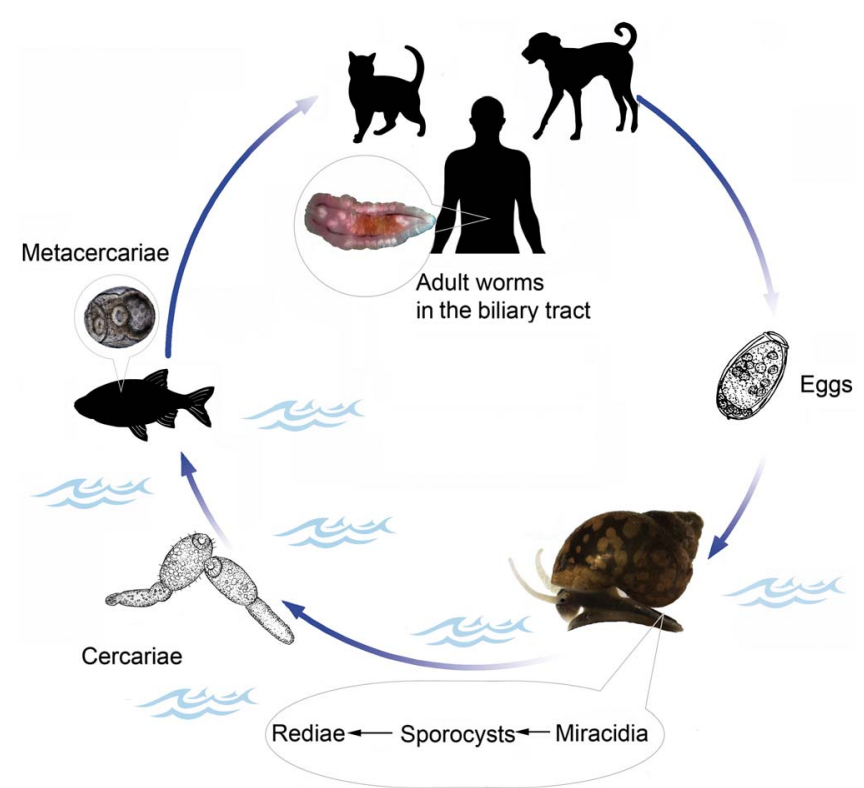

Figure 1. The life cycle of $O$. felineus [15]. The eggs are shed in the biliary tree, enter the intestine, and are passed with feces. They need to be ingested by freshwater gastropod Bithynia snails, the first intermediate host, to develop into sporocysts, rediae, and free-swimming cercariae: the stage infective for the second intermediate host, cyprinid fish. Humans and other fisheating mammals and birds may serve as a definitive host after ingesting infected fish. Upon entering the host body, metacercariae infect the biliary tract of mammals, where they mature into adult worms. Pictures of cercariae and eggs are adapted from [15]; Bithynia snail, metacercaria, and adult O. felineus are original pictures.

inner membrane, then the outer connective tissue capsule, and turn into the next stage, the metacercariae. After 3-4 weeks, metacercariae become invasive and can infect definitive hosts: domestic fish-eating animals (cats, dogs, and pigs), wild carnivores (muskrats, water voles, foxes, bears, and wolves), and humans [14-23]. In the acidic content of the definitive host's stomach, the membrane of the metacercaria is destroyed, and the juveniles migrate along the bile ducts to the liver and gall bladder, where after 25-30 days, the helminth reaches maturation $[14,15]$.

The human infection is most likely due to the consumption of undercooked fish, because sun-dried, lightly salted, and lightly pickled fish are traditional beer snacks (Fig. 2). Furthermore, in the Northern regions of Western Siberia, eating of frozen ribbons of fresh-water whitefish served with spices, so-called stroganina, is a popular tradition [1, 23].

Many authors have demonstrated the presence of metacercariae and adult $O$. felineus worms in the wild. The complete life cycle of $O$. felineus, however, has been demonstrated only by Fedorov, and he has also provided the data about the structure and timing of the development of larval forms [15]. It has been reported that red foxes Vulpes vulpes L. play a certain role in keeping up the natural hot spots of opisthorchiasis in Europe, caused by the 


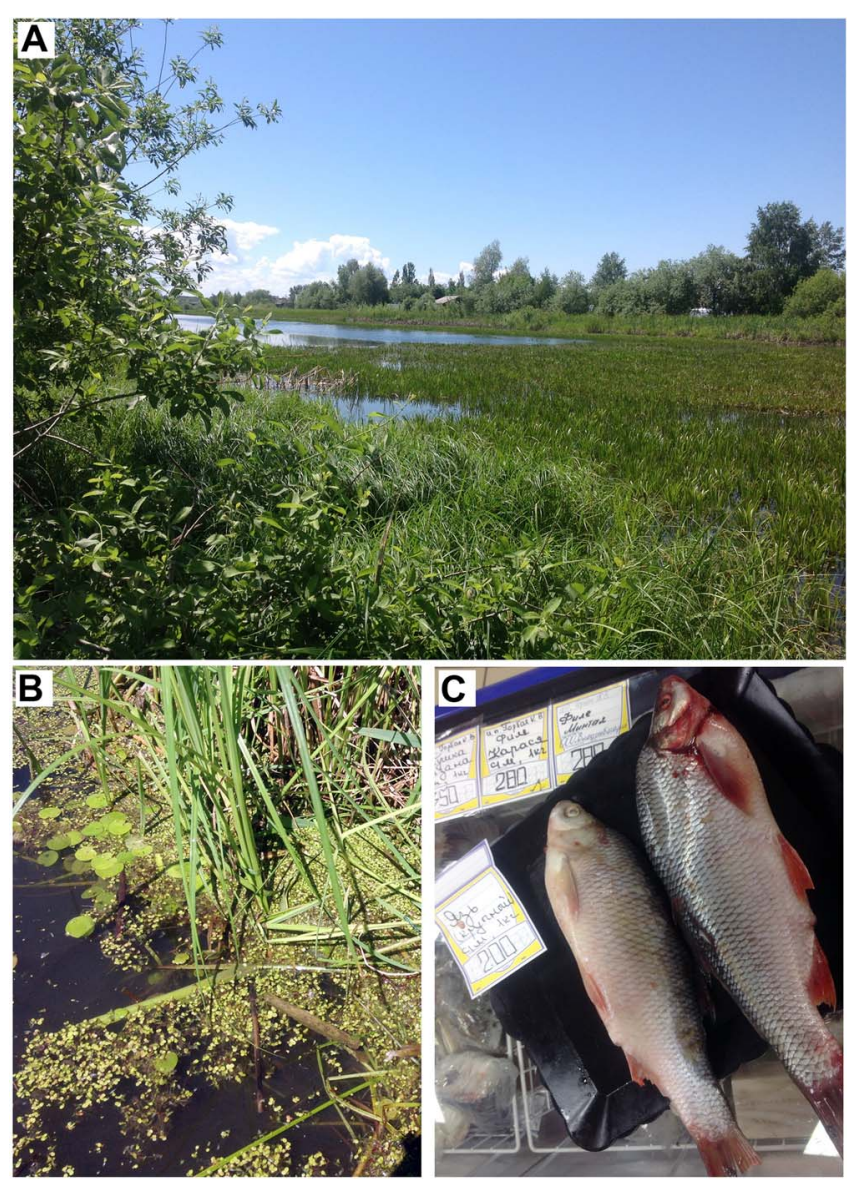

Figure 2. The Maloe lake tributary of the Ob River (Novosibirsk city) and intermediate host for the liver fluke Opisthorchis felineus. (A) Maloe lake. (B) Backwater of Maloe lake, a habitat of Bithynia snails. (C) Fresh ides (L. idus) on ice at a market.

trematode O. felineus [16-21]. The natural host for O. felineus in Russia is thought to be the muskrat Ondatra zibethicus [15], which was introduced in early 1920s. In Western Siberia, the role of muskrats is the primary contribution to the natural causes of the diseases induced by many opisthorchiids like $O$. felineus, $O$. longissimus, and Metorchis bilis [15]. The muskrat leads a semiaquatic lifestyle and settles along the banks of rivers, lakes, and freshwater marshes. It prefers shallow (1-2 $\mathrm{m}$ deep), ponds that do not freeze, with banks that are covered with dense grass [15].

\section{Epidemiology}

The trematode $O$. felineus is an epidemiologically important liver fluke. This causative agent of opisthorchiasis has been detected for some 150 years in cats, dogs, foxes, wild bears, and other animals (the definitive hosts) as well as mollusks and carps (intermediate hosts) in Western and Eastern Europe (Russia, Ukraine, Belorussia, Spain, Portugal, Germany, and Italy) [14, 24, 25]. All these data indicate the breadth of the geographical range of opisthorchiasis and the undoubted participation of animals in the circulation of the pathogen within this area, whereas the human opisthorchiasis has a disjointed local (focal) character. The greatest importance of humans in the circulation of infection is seen in Western Siberia, namely in the Ob-Irtysh basin, as evidenced by the high prevalence of infection in this population (up to $60 \%$ in some rural regions). Thus, most likely, in this region, the participation of humans in the maintenance of the infection is almost certain. Toward the periphery of the basin up to the Arctic Ocean, the percentage of the population living in the area drops to less than one person per square kilometer. Nevertheless, prevalence of the infection among sensitive fish species in this region is almost maximal when compared with other territories and can be up to $100 \%$ (ide). Consequently, the infection circulation becomes more zoonotic in the wild (mainly with participation of domestic and wild animals).

In accordance with the Russian clinical standards, diagnosis of opisthorchiasis is based on the microscopic detection of eggs in stool and/or eggs and adult helminths in bile samples after duodenal intubation [26]. The average opisthorchiasis incidence in Russia between 1991 and 1993 and 2011-2013 was relatively constant (26.5-24.7 cases per 100000 people per year). The incidence of opisthorchiasis in Russia is unevenly distributed across regions. Accordingly, there are hyper-endemic areas (with prevalence rates $>100$ per 100000 people), regions with high prevalence (53-100), regions with average prevalence $(22$ $52)$, and those with low frequency of the disease $(<22)$. The Ob-Irtysh basin in Western Siberia is regarded as a hyper-endemic area. In this region, opisthorchiasis prevalence rates exceed 100 per 100000 people [1]: KhantyMansiiskiy Autonomous Region (600 per 100000 people), Tyumen Region (201 per 100000 people), Tomsk Region (189 per 100000 people), and Novosibirsk Region (127 per 100000 people). In most regions of Russia, Eastern Siberia, and the Far East, only low prevalence is reported (2.5 per 100000 population; reviewed in [1]).

On the other hand, short-term screening programs in some rural regions in this area have revealed that the actual frequency of $O$. felineus infection is much higher [5, 27]. In particular, the prevalence rate in the Tomsk Region has been found to be 18-20\% [27]; Tyumen oblast, 37\%; and Chelyabinsk oblast, $18.6 \%$. According to autopsy data, $18.3-45.0 \%$ of individuals have been found to be infected with opisthorchiasis [5, 28, 29].

\section{The carcinogenic potential of $O$. felineus}

\section{Experimental evidence}

Today, carcinogenesis is thought to be triggered by chronic inflammation and changes in the cellular microenvironment that result in a precancerous state and mutations, which lead to the cell proliferation and malignancy [30]. 

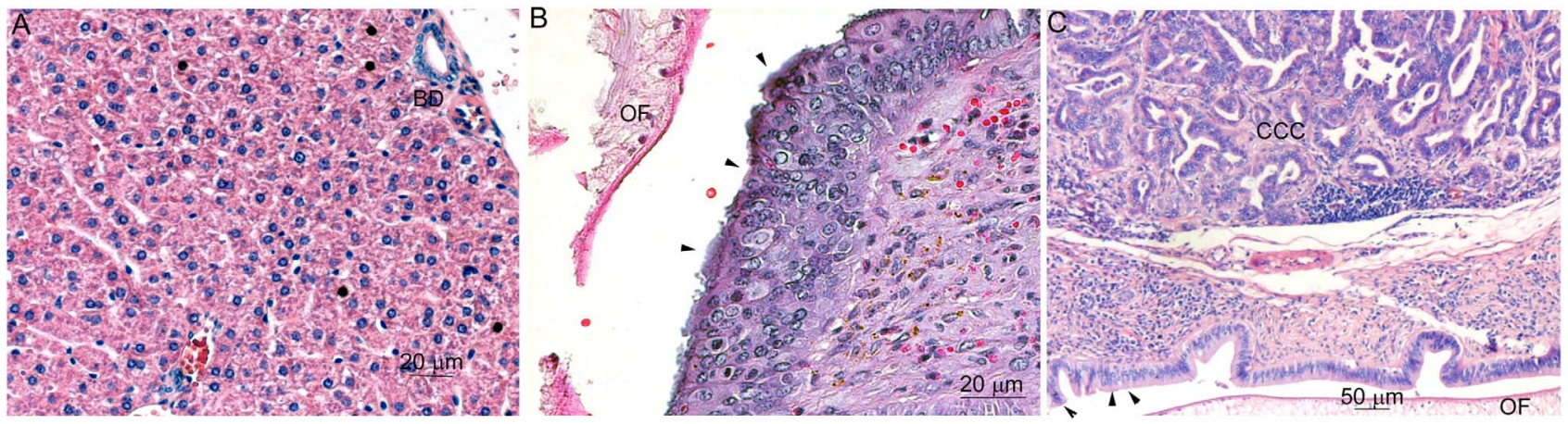

Figure 3. Biliary histological features in the liver biopsies from hamsters with the experimental opisthorchiasis felinea. (A) Intact animal. (B) High-grade biliary neoplasia caused by $O$. felineus three months p.i. (C) Cholangiocarcinoma in the liver of hamster as a result of a combined action of $O$. felineus and dimethylnitrosoamine, three months p.i. BD, bile duct; OF, O. felineus; CCC, cholangiocarcinoma. High-grade biliary neoplasia is indicated with arrows. Pictures 3A and 3C were kindly provided by Maksimova GA.

In subsequent sections of this paper, we present experimental data on the histopathology of opisthorchiasis felinea in hamster liver and $O$. felineus metabolites that can cause mutations in the chromosomal deoxyribonucleic acid (DNA) of host cells. A comparative study of opisthorchiasis felinea and viverrini in a hamster model suggests that the histopathology of $O$. felineus infection involves bile duct dysplasia, periductal fibrosis, and inflammatory infiltration and could be considered to be more severe than that of $O$. viverrini infection [31].

The main histopathological features of $O$. felineus infection in rodents are extensive periductal fibrosis, chronic inflammation, proliferation of the bile duct, bile duct dysplasia, egg granulomas, and biliary intraepithelial neoplasia (BilIN), with grades of $1-3$. BilIN is a precursor lesion of invasive adenocarcinoma in the biliary tract [32-34], and represents a multistep process in carcinogenesis. Biliary intraepithelial neoplasia with grade 1 (BilIN-1) and biliary intraepithelial neoplasia with grade 2 (BilIN-2) lesions have been demonstrated in $O$. felineus-infected hamsters. The totality of biochemical and histopathological changes indicates that $O$. felineus infection in this rodent model induces precancerous lesions conducive to the development of a malignant tumor.

Opisthorchis felineus infection results in CCC in experimental animals if it is accompanied by a chemical carcinogen (e.g., diethylnitrosamine or dimethylnitrosamine). It has been shown that histopathological features of CCC appear in the liver of the hamsters at 18 weeks postinfection [35]. In one study, after 26 weeks, in three of six hamsters, a mass-forming CCC was found, and after 30 weeks postinfection, CCC was detected in all the hamsters [35].

The accumulated data on histopathology in mammals suggest that the carcinogenic potential of $O$. felineus in an experimental model is very similar to the carcinogenicity of $O$. viverrini and $C$. sinensis $[31,35]$. The three species of epidemiologically important opisthorchiids are closely related to one another in terms of the phenotype of adults, the habitats of different life stages, and the genetics.
The liver fluke infections have similar characteristics of the pathogenesis (i.e., mechanism). In addition, the ability to secrete proteins and oxysterol metabolites is also characteristic for liver flukes including $O$. felineus.

The pathogenesis of CCC resulting from the infection with liver flukes is unknown $[13,36]$. Nevertheless, it can be assumed that several important factors are involved. In particular, one of the most important factors is mechanical damage to the wall of the bile duct from suckers of the helminths. When the tissue is damaged, a cascade of immunological reactions is launched both against the destroyed cells and against the helminth antigens and eggs.

Another important factor determining the parasite-host interaction, which probably makes a significant contribution to epithelial neoplasia, is helminth secretory products, consisting of a large number of proteins that induce cell proliferation and inhibit apoptosis [36-38]. Such an effect on the proliferation of epithelial cells of the bile ducts has been demonstrated for 0 . viverrini granulin-1 [36]. The results of CRISPR/Cas9 genome editing of O. viverrini granulin-1 proved the suggestion about its role in the hepatobiliary morbidity of liver fluke infection [37]. Moreover, for another O. viverrini protein, thioredoxin peroxidase $(\mathrm{TpX})$, an immunomodulatory effect has been demonstrated [36]. Particularly, thioredoxin peroxidase can inhibit $\mathrm{T}$ helper 1 (Th1)-type of immune response and activate the Th2 type. A remarkable influence on the host-parasite interplay has also been demonstrated for a defense molecule of Fasciola hepatica helminth. This protein inhibits antigen presentation by macrophages due to its affinity for the membrane of host macrophages [39].

The liver fluke excretory-secretory products (ESPs) stimulate chronic inflammation and proliferation of the bile duct epithelium. Proteomic mass spectrometry of the proteins in O. felineus ESPs has identified 37 proteins [40]. Some of $O$. felineus ESPs are highly immunogenic [41, 42], homologs of host defense molecule (HDM), TpX, and glutathione-S-transferase (GST) have been found in O. felineus ESPs [40]. Moreover, O. felineus ESPs may 
interact with the epithelium and may accumulate inside the cells because they are found in the gallbladder of the hamsters with experimental opisthorchiasis and patients with the infection [41, 42].

In addition, it can be assumed that the specific lowmolecular-weight cholesterol metabolites, which are also found in the helminth secretory product, apparently have oxidative and genotoxic properties, i.e., can damage the DNA of host cells [13, 32]. Such low-molecular-weight metabolites (catechol estrogens and oxysterols) in other species of helminths have been demonstrated, some of which are associated with cancer initiation and are recognized as biological carcinogens. In particular, the trematodes $O$. viverrini, O. felineus, and S. haematobium produce specific oxysterols and catechol estrogens. Oxysterol-like metabolites in the egg and adult worms, and in bile, serum, and urine of $O$. felineus-infected hamsters have been detected by liquid chromatography with mass spectroscopy [32]. Related DNA adducts suggest that infection-associated oxysterols induce chromosomal lesions in host cells [13]. Associations between oxysterols and the initiation and progression of cancer of the colon, lungs, breast, and bile ducts have been proposed [43, 44].

The origin of these metabolites is unknown. It can be assumed that there are two pathways for their synthesis: enzymatic and nonenzymatic. The nonenzymatic pathway is the reaction with the free oxygen and nitrogen species [45]. The enzymatic pathway is the result of the liver fluke xenobiotic metabolism network activity $[46,47]$.

It is generally accepted that CCC tumors in this hamster model occur as a result of a combined action of infection with the liver flukes, O. felineus, O. viverrini, or C. sinensis and exposure to $\mathrm{N}$-nitrosodimethylamine [35]. In contrast, a series of studies on the pathogenesis of opisthorchiasis felinea has revealed enhanced expression of p53, and the presence of intraepithelial bile duct neoplasia $[32,35]$. Recent studies have also shown that epithelial-tomesenchymal transition (EMT) is involved in chronic opisthorchiasis in rodents. At 22 weeks of chronic opisthorchiasis in hamsters, processes of EMT intensify in the liver [48]. The increased expression of markers of the SMAD signaling pathway and enhanced accumulation of $\alpha$-smooth muscle actin ( $\alpha$-SMA) protein have been shown by immunohistochemistry. The elevated messenger ribonucleic acid (mRNA) expression of transforming growth factor beta 1 (Tgfb1), annexin A1 (Anxa1), exostosin glycosyltransferase 1 (Ext1), and keratin 7 (Krt $\left.{ }^{r}\right)$ genes has been demonstrated in the liver of $O$. felineus-infected hamsters [48]. Taken together, these data indicate that precancerous changes can occur in hamsters without exposure to an exogenous carcinogen such as N-nitrosodimethylamine (NDMA). These processes cause damage to genetic material, with the mutations being fixed during DNA replication. Eventually, accumulation of mutations might lead to malignant transformation of cholangiocytes and progression to CCC [13].

1. The link between O. felineus infection and CCC in humans
The most important step in the validation of results obtained in a study on the carcinogenic potential of $O$. felineus in an experimental model is analysis of the association of opisthorchiasis felinea and CCC in humans. Given that there are no systemic epidemiological and clinical programs for the control of opisthorchiasis in Russia, the solution to this problem remains difficult. Undoubtedly, the similarity in clinical manifestations, the disorders caused by $O$. felineus and O. viverrini $[28,29]$, and the data on the pathogenesis of these two helminthiases acquired in experimental opisthorchiasis models $[1,31,32,34]$ are suggestive of a rather high probability of $O$. felineus involvement in the induction of these tumors. Here, we briefly discuss the data published in the Russian medical literature on the complications associated with opisthorchiasis as well as the data from hospitals and morgues on autopsy results over a long period. Unfortunately, the data on liver cancer prevalence have certain drawbacks, in particular, the performed examinations and criteria for selection of control groups are insufficiently detailed. Nonetheless, in the absence of other epidemiological data, the number of cases recorded in hospitals during a given period is also valuable information, of course, with a disclaimer that this is simple recording of the number of cases rather than full-fledged epidemiological studies.

In general, it is reported that the incidence of malignant tumors, in particular, primary liver cancer is significantly higher in regions with high prevalence of opisthorchiasis. In a number of regions of Western Siberia in the period 1962-1971, the prevalence of primary liver cancer was 9.4 cases per 100000 people $[49,50]$ (reviewed in [7]). In the period 1990-2001, liver cancer ranked seventh among the most common cancers registered in the Tomsk region $(3.9 \%)$. The prevalence of this type of cancer in men in this period was 11.2 cases per 100000 people [51]. This is almost 2.5 -fold more than the average prevalence rate of liver cancer in the Russian Federation in 2012, according to the official statistics [51, 52]. For comparison, in Moscow and Moscow region (non-endemic areas of opisthorchiasis) the prevalence of primary liver cancer was 1.77 and 2.3 cases per 100000 people, respectively [53]. The incidence of primary liver cancer in these regions is not correlated with alcohol consumption, in particular Moscow region was in the top three for the consumption of strong alcoholic drinks (2016-2017), whereas Tomsk and Tyumen regions (hyperendemic) were with low or moderate scores [54].

In the Russian medical literature, there is information indicating a link between $O$. felineus infection and liver cancer in the population of Western Siberia. Thus, during analysis of the results of more than 2250 autopsies conducted during 37 years in the city morgue of Tobolsk, it was found that in $45 \%$ of the cases, infection with opisthorchiasis was present. Primary liver cancer has been diagnosed in $8.7 \%$ of the infected patients [55]. According to the Tomsk City Morgue, among persons with a confirmed diagnosis of chronic opisthorchiasis [ 350 autopsies], CCC cases have been registered in $5 \%$ of the group [28, 29]. Furthermore, according to the information from the Tomsk Hepatology Center, $\sim 3 \%$ of patients with chronic opisthorchiasis have 
been operated on for biliary tract cancer and pancreatic cancer [29].

An interesting study has been conducted in another region of Western Siberia: the Tyumen region. There, the prevalence rates of various types of liver cancer in opisthorchiasis-positive (target group) and negative individuals (control group) were determined. It was established that CCC is most common in opisthorchiasis: according to the results of histological analysis in the target group, this diagnosis was made in $77 \%$ of the cases. Hepatocellular carcinoma (HCC) in the same group of patients was detected in $19 \%$ of cases, and $4 \%$ of patients had other types of malignant liver tumors. In the control group, CCC occurred in $34 \%$ of patients, hepatocellular carcinoma in $56 \%$ of cases, and other liver tumors in $10 \%$ [49].

In agreement with these results, the data obtained in the Tyumen region - after autopsies of 44 individuals with confirmed primary liver cancer - have revealed that CCC was present in almost $80 \%$ of cases. It is important to note that O. felineus infection was confirmed in 42 of 44 cases in that study (reviewed in $[7,56])$. Thus, in endemic regions, malignant tumors of the biliary tract are some of the most common types of liver cancer. It is noteworthy that in the presence of $O$. felineus infection, the prevalence of $\mathrm{CCC}$ exceeds that of other types of liver cancer by the factor of four (or by 400\%). Recently, two cases of CCC associated with chronic opisthorchiasis were reported. Both cases presented with nonspecific symptoms and severe pathological complications. Both patients were living in endemic areas [26].

In non-endemic areas of opisthorchiasis in Russia, the proportion of CCC does not exceed $15 \%$ of all cases of liver cancer. For example, according to generalized data accumulated at the Blokhin Russian Cancer Research Center (Moscow), CCC is responsible for $14.9 \%$, and hepatocellular cancer for $85.1 \%$ of liver cancer cases [57]. In general, data on the prevalence of CCC in the endemic and non-endemic regions well match the general epidemiology of this type of cancer in other endemic and non-endemic regions of the world $[9,58]$.

\section{Prevention}

Prevention of opisthorchiasis is based primarily on the inactivation of $O$. felineus larvae in the fish by proper cooking, the disease diagnostics, and effective treatment of the patients. Preventive measures include (i) protection of reservoirs from eggs of the parasite, (ii) the inactivation of metacercariae in relevant species of fish, and (iii) sanitation/educational programs for the population.

In Russia, where the rate of infection with opisthorchiids among fish is high, standards have been developed to combat the contamination of fish by larvae of epidemiologically important liver flukes including O. felineus, M. bilis, and C. sinensis [59]. These standards stipulate first and foremost salting, freezing, and heat treatment. Salting should be done by means of sufficient amounts of salt at a temperature of $1-20{ }^{\circ} \mathrm{C}$, when the mass fraction of salt in fish is
14\%. Duration of salting should be 10 days for bleak Alburnus alburnus, gudgeon Gobio gobio, minnow Phoxinus percnurus, and sunbleak Leucaspius delineatus; 21 days for roach $R$. rutilus, dace $L$. leuciscus, common rudd Scardinius erythrophthalmus, small common bream Abramis brama, and ide Leuciscus idus; and 40 days for large common bream and ide [60].

Standards provide for the resistance of the metacercariae to low temperatures. Fish should be frozen under the following conditions: $-40{ }^{\circ} \mathrm{C}$ for $7 \mathrm{~h} ;-35{ }^{\circ} \mathrm{C}$ for $14 \mathrm{~h}$; $-28{ }^{\circ} \mathrm{C}$ for $32 \mathrm{~h} \mathrm{[58].} \mathrm{Hot} \mathrm{and} \mathrm{cold} \mathrm{smoking,} \mathrm{dry} \mathrm{curing,}$ and drying performed properly according to the official standards disinfect all kinds of fish except ide. Unfortunately, metacercariae inactivation measures are difficult to use when fish is processed at home. Thus, it is almost impossible to provide the necessary conditions for freezing in home refrigerators [61]. Traditional drying of fish and cold smoking do not inactivate parasite larvae [62]. Modern methods have been developed to inactivate the larvae, for example, the high efficiency of the use of microwave ovens in the disinfection of large fish has been demonstrated as compared with other methods [63].

It should be noted that in terms of control of opisthorchiasis, educational programs for the population of Western Siberia are not being actively pursued [64]. Thus, a significant part of the population of the north Tyumen region thinks that raw fish is healthy food (32\% of the surveyed population) and less than a half of the population know about liver fluke infections ( $45.9 \%$ of the population). The infection prevalence of the population in this region is $\sim 40 \%$. It is noteworthy that newcomers also get rapidly infected: in the first year after arrival: $2 \%$, within five years: $10 \%$, and within 10 years, $25 \%$ of newcomers become infected. During the first year after arrival, only $8 \%$ of newcomers consider raw fish a healthy food, but after 5-10 years, their number significantly increases and constitutes $17 \%$. These data point to successful adaptation of the settlers to the peculiarities of local nutrition and their eating habits in the aboriginal style.

Only $39 \%$ of healthcare professionals in the region know how to use the technology of pickling, which guarantees disinfection of fish. It should be noted that drying in this region is one of the most common ways of processing of fish. Only $9 \%$ of physicians, $27 \%$ of epidemiologists, and $11.5 \%$ of other medical professionals in the region have mastery of the proper fish drying technology that guarantees decontamination [64]. Accordingly, the level of knowledge about the invasion of fish by opisthorchiids and about the dangers of contamination is low even among people with medical education in this region. Apparently, all these factors in combination lead to the high prevalence of infection with opisthorchiids in this region.

\section{Conclusion}

The carcinogenic potential of the trematode $O$. felineus appears to be poorly studied as compared to that of closely related liver flukes $O$. viverrini and $C$. sinensis. 
Nevertheless, O. felineus habitat covers vast areas in Europe and Asia, and opisthorchiasis outbreaks caused by this helminth are expectable in many countries. Thus, the opisthorchiasis caused by $O$. felineus becomes a large-scale problem, outside the scope of health care and public health measures in individual regions.

Opisthorchiasis is a common human helminthiasis in Russia. In Thailand and Korea, the consumption of raw fish is traditionally widespread; in Russia, the tradition of eating raw fish is not as popular as eating sun-dried and lightly salted fish, which are apparently infected with metacercaria of the parasite. In contrast to Thailand (the endemic area for O. viverrini), where CCC is a predominant type of cancer, primary liver cancer is not the leading cancer type in Russia. There are probably some additional factors that lead to carcinogenesis in humans. Apparently, one of the key factors, as shown by experiments on model animals, is the presence of weak carcinogens in food, such as nitrosamines, which act as initiators of carcinogenesis. Hence, this chronic infection is expected to promote the initiation of biliary cancers. There are some other factors that are relative risks and cofactors for CCC formation. In Thailand and the Republic of Korea, the highest relative risk has been adjusted for alcohol consumption, smoking, and physiological characteristics such as sex and age [7]. These factors should be taken into consideration in the areas endemic for opisthorchiasis felinea as well.

To date, the IARC has classified $O$. felineus infection as a Group 3 carcinogen, i.e. there is insufficient evidence yet for its classification as carcinogenic, unlike the situation with the closely related liver flukes $O$. viverrini and C. sinensis, and the blood fluke S. haematobium which are group 1 carcinogens: definitely carcinogenic to humans. For IARC approval O. felineus as Group 1 carcinogen, carcinogenic activity should be demonstrated in animal and human studies.

The accumulated data on the histopathology in animal models show that the carcinogenic activity of $O$. felineus in an experimental model is very similar to the carcinogenicity of $O$. viverrini and C. sinensis. All species of epidemiologically important opisthorchiids are similar to one another in terms of the phenotype of adults, the habitats of different life stages, and the genetics. The liver fluke infections share nearly the same pathogenesis and host liver histopathology. In addition, the ability to secrete proteins and oxysterol metabolites is characteristic of liver flukes including O. felineus.

Large-scale studies on the association of opisthorchiasis felinea with CCC in humans have not been conducted, but such information might be found at least partly in the published literature and in hospital records. The consolidated data show that the prevalence of primary liver cancer in Western Siberian regions, the world's largest endemic area of opisthorchiasis felinea, is considerably higher as compared with the non-endemic regions. Nevertheless, the presented data were obtained by separate groups of researchers and cannot present a comprehensive picture of the association of opisthorchiasis and liver cancer. This task requires largescale epidemiological studies that take advantage of the expertise gained in similar studies in other countries [9].

\section{Abbreviations used in the text and figures}

$\alpha$-SMA $\quad \alpha$-smooth muscle actin

Anxa1 Annexin A1

BD Bile duct

BilIN-1 Biliary intraepithelial neoplasia with grade 1

BilIN-2 Biliary intraepithelial neoplasia with grade 2

CCC Cholangiocarcinoma

DNA Deoxyribonucleic acid

EMT Epithelial-to-mesenchymal transition

ESPs Excretory-secretory products

Ext1 Exostosin glycosyltransferase 1

GST Glutathione-S-transferase

HCC Hepatocellular carcinoma

HDM Host defense molecule

IARC International Agency for Research on Cancer

Krt7 $\quad$ Keratin 7

mRNA Messenger ribonucleic acid

NDMA N-nitrosodimethylamine

OF O. felineus

p.i. Postinfection

Tgfb1 Transforming growth factor beta 1

$\mathrm{TpX} \quad$ Thioredoxin peroxidase

\section{Acknowledgments}

Figure $3 \mathrm{~A}$ and $\mathrm{C}$ were kindly provided by Galina A Maksimova (ICG SB RAS, Novosibirsk, Russia). Authors acknowledge the support of members of the TOPIC [Tomsk OPIsthorchiasis Consortium]. The English language was corrected and certified by shevchuk-editing. com.

\section{Funding}

This work was supported financially by the Russian Foundation for Basic Research, https://www.rfbr.ru/rffi/ eng [No. 18-04-00417A [VAM] and the state project of the Institute of Cytology and Genetics, the Siberian Branch of the Russian Academy of Sciences [project No. 0324-20190041] (MYP, VAM). The sponsor had no role in this study such as study design, data collection, or decision to publish.

\section{Conflict of interest}

The authors declare that they have no competing interests. 


\section{References}

1. Pakharukova MY, Mordvinov VA (2016), The liver fluke Opisthorchis felineus: biology, epidemiology, and carcinogenic potential. Trans R Soc Trop Med Hyg 110, 28-36. https://doi.org/10.1093/trstmh/trv085.

2. Shain AA (1974), Symptomatology of primary liver cancer. Vopr Onkol 20, 9, 50-56.

3. Blokhin NN, Klimenkov AA, Itin AV, Komov DV, Assekritova IV (1978), Primary cancer of the liver according to the materials of the Scientific Oncological Center of the AMS USSR. Vestn Akad Med Nauk SSSR 5, 3-9.

4. Beer SA (2005), Biology of the agent of opisthorchiasis, KMK, Moscow. ISBN 5-87317-204-8.

5. Zubov NA, Belikov ES, Zaŭtseva LN (1989), Opisthorchiasis in Tobolsk based on 1950-1987 autopsy data. Med Parazitol [Mosk] 5, 10-13.

6. Tselepatiotis E, Mantadakis E, Papoulis S, Vassalou E, Kotsakis P, Samonis G (2003), A case of Opisthorchis felineus infestation in a pilot from Greece. Infection 31, 6, 430-432. https://doi.org/10.1007/s15010-003-3225-z.

7. IARC Working Group on the Evaluation of Carcinogenic Risks to Humans (2012), Biological agents. Volume 100 B. A review of human carcinogens. IARC Monogr Eval Carcinog Risks Hum, 100, Pt B, 1-441.

8. Sithithaworn P, Yongvanit P, Duenngai K, Kiatsopit N, Pairojkul C (2014), Roles of liver fluke infection as risk factor for cholangiocarcinoma. J Hepatobiliary Pancreat Sci 21, 301-308. https://doi.org/10.1002/jhbp.62.

9. Sripa B, Tangkawattana S, Laha T, Kaewkes S, Mallory FF, Smith JF, Wilcox BA (2015), Toward integrated opisthorchiasis control in northeast Thailand: the Lawa project. Acta Trop 141, 361-367. https://doi.org/10.1016/j.actatropica. 2014.07.017.

10. Gibson JB (1978), Histological typing of tumours of the liver, biliary tract, and pancreas, in: Gibson JB, Sobin LH (Eds.), International histological classification of tumours no. 20, World Health Organization Publications Centre, Geneva. ISBN 3-540-60280-1

11. Pellino A, Loupakis F, Cadamuro M, Dadduzio V, Fassan M, Guido M, Cillo U, Indraccolo S, Fabris L (2018), Precision medicine in cholangiocarcinoma. Transl Gastroenterol Hepatol 3, 40. https://doi.org/10.21037/tgh.2018.07.02.

12. Blechacz B, Gores GJ (2008), Cholangiocarcinoma: advances on pathogenesis, diagnosis, and treatment. Hepatology 48, 308-321. https://doi.org/10.1002/hep.22310.

13. Brindley PJ, Costa JMCD, Sripa B (2015), Why does infection with some helminths cause cancer? Trends Cancer 1, 3, 174-182. https://doi.org/10.1016/j.trecan.2015.08.011.

14. Fedorov KP, Naumov VA, Kuznetsova VG, Belov GF (2002), Some real problems of human opisthorchiasis. Med Parazitol (Mosk) 3, 7-9. Russian.

15. Fedorov KP (1968), The role of muskrat in the distribution of opisthorchiasis in the north of the Tomsk region. Parazitologiia 11, 3, 258-259.

16. Segovia JM, Torres J, Miquel J (2004), Helminth parasites of the red fox (Vulpes vulpes L., 1758) in the Iberian Peninsula: an ecological study. Acta Parasitol 49, 67-79.

17. Segovia JM, Torres J, Miquel J (2002), The red fox, Vulpes vulpes L., as a potential reservoir of zoonotic flukes in the Iberian Peninsula. Acta Parasitol 47, 163-166.

18. Rajkovic-Janje R, Marinculic A, Bosnic S, Benic M, Vinkovic B, Mihaljevic Z (2002), Prevalence and seasonal distribution of helminth parasites in red foxes (Vulpes vulpes) from the Zagreb County (Croatia). Z Jagdwiss 48, 151-160.

19. Shimalov VV, Shimalov VT, Shimalov AV (2000), Helminth fauna of otter (Lutra lutra Linnaeus, 1758) in Belorussian
Polesie. Parasitol Res 86, 6, 528. https://doi.org/10.1007/ s004360050708.

20. Shimalov VV, Shimalov VT (2001), Helminth fauna of the American mink (Mustela vison Schreber, 1777) in Belorussion Polesie. Parasitol Res 87, 886-887. https://doi.org/ $10.1007 / \mathrm{s} 004360100461$.

21. Shimalov VV, Shimalov VT (2003), Helminth fauna of the red fox (Vulpes vulpes Linnaeus, 1758) in southern Belarus. Parasitol Res 89, 77-78. https://doi.org/10.1007/s00436002-0701-9.

22. Mordvinov VA, Yurlova NI, Ogorodova LM, Katokhin AV (2012), Opisthorchis felineus and Metorchis bilis are the main agents of liver fluke infection of humans in Russia. Parasitol Int 61, 25-31. https://doi.org/10.1016/j.parint.2011.07.021.

23. Mordvinov VA, Furman DP (2010), The Digenea parasite Opisthorchis felineus: a target for the discovery and development of novel drugs. Infect Disord Drug Targets 10, 385401. https://doi.org/10.2174/187152610793180858.

24. Pozio E, Armignacco O, Ferri F, Gomez Morales MA (2013), Opisthorchis felineus, an emerging infection in Italy and its implication for the European Union. Acta Trop 126, 54-62. https://doi.org/10.1016/j.actatropica.2013.01.005.

25. Akuffo H, Linder E, Ljungstrom I, Wahlgren M (2002), Parasites of the colder climates, CRC Press, London, New York. ISBN 9780429218774.

26. Kovshirina YV, Fedorova OS, Vtorushin SV, Kovshirina AE, Ivanov SD, Chizhikov AV, Onishchenko SV, Ogorodova LM, Odermatt P (2019), Case Report: two cases of cholangiocarcinoma in patients with Opisthorchis felineus infection in Western Siberia, Russian Federation. Am J Trop Med Hyg 100, 3, 599-603. https://doi.org/10.4269/ajtmh.18-0652.

27. Ilyinskikh EN, Novitsky VV, Ilyinskikh NN, Lepyokhin AV (2007), Opisthorchis felineus (Rivolta, 1884) and Metorchis bilis (Braun, 1890) infections in population of some regions of the Ob river basin. Parasitol (Mosk) 41, 55-64. [in Russian].

28. Brazhnikova NA, Tolkaeva MV (2002), Cancer of liver, biliary tracts and pancreas at chronic opisthorchiasis. Bull Sib Med 2, 71-77. [in Russian].

29. Brazhnikova NA, Tskhai VF (2009), Opisthorchiasis-induced suppurative cholangitis. Annaly hirurgicheskoy gepatologii [Ann Surg Hepatol, Mosk] 14, 28-33. [in Russian].

30. Brücher BL, Jamall IS (2014), Epistemology of the origin of cancer: a new paradigm. BMC Cancer 10, 14, 331. https://doi.org/10.1186/1471-2407-14-331.

31. Lvova MN, Tangkawattana S, Balthaisong S, Katokhin AV, Mordvinov VA, Sripa B (2012), Comparative histopathology of Opisthorchis felineus and Opisthorchis viverrini in a hamster model: an implication of high pathogenicity of the European liver fluke. Parasitol Int 61, 167-172. https://doi. org/10.1016/j.parint.2011.08.005.

32. Gouveia MJ, Pakharukova MY, Laha T, Sripa B, Maksimova GA, Rinaldi G, Brindley PJ, Mordvinov VA, Amaro T, Santos LL, Correia da Costa JM, Vale N (2017), Infection with Opisthorchis felineus induces intraepithelial neoplasia of the biliary tract in a rodent model. Carcinogenesis 38, 929937. https://doi.org/10.1093/carcin/bgx042.

33. Zen Y, Adsay NV, Bardadin K, Colombari R, Ferrell L, Haga H, Hong SM, Hytiroglou P, Klöppel G, Lauwers GY, van Leeuwen DJ, Notohara K, Oshima K, Quaglia A, Sasaki M, Sessa F, Suriawinata A, Tsui W, Atomi Y, Nakanuma Y (2007), Biliary intraepithelial neoplasia: an international interobserver agreement study and proposal for diagnostic criteria. Mod Pathol 20, 701-709. https://doi.org/10.1038/ modpathol.3800788.

34. Tsokos CG, Krings G, Yilmaz F, Ferrell LD, Gill RM (2016), Proliferative index facilitates distinction between benign biliary lesions and intrahepatic cholangiocarcinoma. Hum 
Pathol 57, 61-67. https://doi.org/10.1016/j.humpath.2016. 06.019.

35. Maksimova GA, Pakharukova MY, Kashina EV, Zhukova NA, Kovner AV, Lvova MN, Katokhin AV, Tolstikova TG, Sripa B, Mordvinov VA (2017), Effect of Opisthorchis felineus infection and dimethylnitrosamine administration on the induction of cholangiocarcinoma in Syrian hamsters. Parasitol Int 66, 458-463. https://doi.org/10.1016/ j.parint.2015.10.002.

36. Sripa B, Brindley PJ, Mulvenna J, Laha T, Smout MJ, Mairiang E, Bethony JM, Loukas A (2012), The tumorigenic liver fluke Opisthorchis viverrini multiple pathways to cancer. Trends Parasitol 28, 395-407. https://doi.org/ 10.1016/j.pt.2012.07.006.

37. Arunsan P, Ittiprasert W, Smout MJ, Cochran CJ, Mann VH, Chaiyadet S, Karinshak SE, Sripa B, Young ND, Sotillo J, Loukas A, Brindley PJ, Laha T (2019), Programmed knockout mutation of liver fluke granulin attenuates virulence of infection-induced hepatobiliary morbidity. Elife 8, e41463, https://doi.org/10.7554/eLife.41463.

38. Chaiyadet S, Sotillo J, Smout M, Cantacessi C, Jones MK, Johnson MS, Turnbull L, Whitchurch CB, Potriquet J, Laohaviroj M, Mulvenna J, Brindley PJ, Bethony JM, Laha T, Sripa B, Loukas A (2015), Carcinogenic liver fluke secretes extracellular vesicles that promote cholangiocytes to adopt a tumorigenic phenotype. J Infect Dis 212, 1636-1645. https://doi.org/10.1093/infdis/jiv291.

39. Robinson MW, Alvarado R, To J, Hutchinson AT, Dowdell SN, Lund M, Turnbull L, Whitchurch CB, O'Brien BA, Dalton JP, Donnelly S (2012), A helminth cathelicidin-like protein suppresses antigen processing and presentation in macrophages via inhibition of lysosomal vATPase. FASEB J 26, 11, 4614-4627. https://doi.org/10.1096/fj.12-213876.

40. Lvova MN, Duzhak TG, Tsentalovich IuP, Katokhin AV, Mordvinov VA (2014), Secretome of the adult liver fluke Opisthorchis felineus. Parazitologiia 48, 3, 169-184.

41. Pakharukova MY, Kovner AV, Trigolubov AN, Fedin EN, Mikhailova ES, Shtofin SG, Autenshlyus AI, Mordvinov VA (2017), Mechanisms of trematodiases pathogenicity: the presence of the secretory proteins from the liver fluke Opisthorchis felineus in the gallbladder tissues of the patients with chronic opisthorchiasis. Vavilov J Genet Breed 21, 3, 312-316. https://doi.org/10.18699/VJ16.17-o.

42. Petrenko VA, Pakharukova MY, Kovner AV, Lvova MN, Lyakhovich VV, Mordvinov VA (2017), Secretion of thioredoxin peroxidase protein of cat liver fluke Opisthorchis felineus during modeling of experimental opisthorchiasis. Bull Exp Biol Med 162, 6, 773-776. https://doi.org/10.1007/ s10517-017-3710-5.

43. Cavalieri EL, Rogan EG (2010), Depurinating estrogen-DNA adducts in the etiology and prevention of breast and other human cancers. Future Oncol 6, 75-91. https://doi.org/ 10.2217 fon.09.137.

44. Jaworski CJ, Moreira E, Li A, Lee R, Rodriguez IR (2001), A family of 12 human gene containing oxysterol-binding domains. Genomics 78, 185-196. https://doi.org/10.1006/ geno.2001.6663.

45. Yongvanit P, Pinlaor S, Bartsch H (2012), Oxidative and nitrative DNA damage: key events in opisthorchiasis-induced carcinogenesis. Parasitol Int 61, 130-135. https://doi.org/ 10.1016/j.parint.2011.06.011.

46. Pakharukova MY, Ershov NI, Vorontsova EV, Katokhin AV, Merkulova TI, Mordvinov VA (2012), Cytochrome P450 in fluke Opisthorchis felineus: identification and characterization. Mol Biochem Parasitol 181, 2, 190-194. https://doi. org/10.1016/j.molbiopara.2011.11.005.
47. Pakharukova MY, Vavilin VA, Sripa B, Laha T, Brindley PJ, Mordvinov VA (2015), Functional analysis of the unique cytochrome $\mathrm{P} 450$ of the liver fluke Opisthorchis felineus. PLoS Negl Trop Dis 9, e0004258. https://doi.org/10.1371/ journal.pntd.0004258.

48. Kovner AV, Maksimova GA, Mordvinov VA (2016), Role of epithelial-to-mesenchymal transition in liver associated with Opisthorchis felineus infection, in: Abstractbook of the International Symposium Systems Biology and Biomedicine (SBIOMED-2016), ICG SB RAS, Novosibirsk. ISBN 978-591291-025-8.

49. Chaklin AV, Shain AA (1976), Results of epidemiological studies of primary cancer of the liver in the Tiumen district. Vopr Onkol 22, 76-82.

50. Shain AA (1971), Opisthorchiasis and hepatic cancer among the population of the Hanty-Mansy National District. Vopr Onkol 17, 34-39.

51. Pisareva LF, Choynzonov YL, Boyarkina AP (2003), Peculiarities of cancer incidence in Tomsk region population (1990-2001). Bull Sib Med 4, 86-97.

52. Caprin AD, Starinsky VV, Petrova GV (2013), Status of cancer care in Russia in 2012, Moscow, FGBI MNIOI them, The Russian Ministry of Health, Herzen. ISBN 978-5-85502187-5.

53. Caprin AD, Starinsky VV, Petrova GV (2018), Malignant tumors in Russia in 2017 (incidence and mortality), Moscow, FGBI MNIOI them, The Russian Ministry of Health, Herzen. ISBN 978-5-85502-243-8.

54. Popova AY (2017), On the state of sanitary and epidemiological well-being of the population in the Russian Federation in 2016: state report, Federal Service for Supervision of Consumer Rights Protection and Human Welfare, Moscow. https:// www.rospotrebnadzor.ru/upload/iblock/0b3/gosudarstvennyy-doklad-2016.pdf.

55. Zubov NA, Amelin SP, Tretiakov AM (1991), Outcomes of injuries of the liver and gallbladder in patients with opisthorchiasis. Khirurgiia (Mosk) 2, 63-66.

56. Glumov VI, Kotrikov VV, Tretiakova NA (1974), Pathogenesis and morphology of primary liver cancer developing on a background of opisthorchiasis. Vopr Onkol 20, 46-50. [in Russian].

57. Patiutko II, Sagaidak IV, Chuchuev ES (2008), Surgical treatment of primary liver cancer. Pract Onkol 9, 197-201.

58. Parkin DM, Whelan SL, Ferlay J, Teppo L, Thomas DB (2002), Cancer incidence in five continents, Vol. VIII, International Agency for Research on Cancer, Lyon. ISBN 978-92-832-2155-5.

59. Popova AY (2015), Prevention of parasitic diseases on the territory of the Russian Federation [Sanitary rules and norms SanPiN 3.2.3215-14] Information and publishing centre of the Ministry of Health, Moscow. http://docs.cntd.ru/document/ 420233490.

60. Pelgunov AN, Kazakov BE, Fedorov KP (2006), Parasitic fauna in the fish of the Ob River basin, in: Pavlov D, Mochek $\mathrm{AD}$ (Eds.), Fish ecology in the Ob-Irtysh river Basin, KMK, Moscow. ISBN 5-87317-300-1

61. Fattakhov RG (2002), Fish infection with Opisthorchis larvae in Russia and some contiguous countries (by the materials of the "Cadaster of opisthorchis infection foci in Russia in 1994"]. Med Parazitol (Mosk) 1, 25-27. Russian.

62. Sidorov EG, Beliakova IuV, Kukashev DSh (1991), An assessment of the opisthorchiasis situation in the area of Irtysh-Karaganda Canal. Med Parazitol (Mosk) 3, 40-42. Russian. 
63. Pelgunov AN, Ryabov IN, Filippova AYu (2005), The use of microwave ovens for the prevention of opisthorchiasis. Med Parazitol (Mosk) 3, 42-45. [in Russian].
64. Pustovalova Via (2002), Features of the sanitary-educational work in opisthorchiasis under conditions of migration pressure in an endemic region. Med Parazitol (Mosk) 3, 10-13. Russian.

Cite this article as: Pakharukova MY, Correia da Costa JM \& Mordvinov VA, 2019. The liver fluke Opisthorchis felineus as a group III or group I carcinogen. 4open, 2, 23. 